\title{
Accuracy of liquid-based brush cytology and HPV detection for the diagnosis and management of patients with oropharyngeal and oral cancer
}

\author{
Paola Castillo ${ }^{1,2,3} \cdot$ Jorge de la Oliva $^{1,2} \cdot$ Silvia Alos $^{1} \cdot$ Francisco Perez $^{1} \cdot$ Naiara Vega $^{1} \cdot$ Isabel Vilaseca $^{2,3,4,6}$. \\ Carles Marti ${ }^{5} \cdot$ Ada Ferrer $^{5} \cdot$ Llucia Alos ${ }^{1,2,3}$
}

Received: 1 August 2021 / Accepted: 13 October 2021 / Published online: 27 November 2021

(c) The Author(s) 2021

\begin{abstract}
Objectives This study aims to evaluate the usefulness of liquid-based brush cytology for malignancy diagnosis and HPV detection in patients with suspected oropharyngeal and oral carcinomas, as well as for the diagnosis of tumoral persistence after treatment.

Material and methods Seventy-five patients with suspicion of squamous cell carcinoma of the oropharynx or oral cavity were included. Two different study groups were analyzed according to the date of the sample collection: (1) during the first endoscopy exploration and (2) in the first control endoscopy after treatment for squamous cell carcinoma. Sensitivity, specificity, positive predictive value, negative predictive value, and accuracy for malignancy diagnosis as well as for HPV-DNA detection on brush cytologies were assessed.

Results Before treatment, the brush cytology showed a sensitivity of $88 \%$, specificity of $100 \%$, and accuracy of $88 \%$. After treatment, it showed a sensitivity of $71 \%$, specificity of $77 \%$, and accuracy of $75 \%$. HPV-DNA detection in cytology samples showed a sensitivity of $85 \%$, specificity of $100 \%$, and accuracy of $91 \%$ before treatment and an accuracy of $100 \%$ after treatment.

Conclusions Liquid-based brush cytology showed good accuracy for diagnosis of oropharyngeal and oral squamous cell carcinoma before treatment, but its value decreases after treatment. Nevertheless, it is useful for HPV-DNA detection, as well as to monitor the patients after treatment.

Clinical relevance Brush cytology samples are reliable for the detection of HPV-DNA before and after treatment and may be a useful method to incorporate in the HPV testing guidelines.
\end{abstract}

Keywords High-risk human papillomavirus $\cdot$ Cytology cytobrush $\cdot$ Squamous cell carcinoma $\cdot$ p16 $\cdot$ Oropharyngeal carcinoma Oral carcinoma

Paola Castillo and Jorge de la Oliva share first authorship.

Llucia Alos

lalos@clinic.cat

1 Department of Pathology, Hospital Clínic, Villarroel, 170, 08036 Barcelona, Spain

2 School of Medicine, Universitat de Barcelona, Barcelona, Spain

3 Institut dInvestigacions Biomèdiques August Pi I Sunyer, IDIBAPS, Barcelona, Spain
4 Department of Otorhinolaryngology, Hospital Clínic, Barcelona, Spain

5 Department of Maxillofacial Surgery, Hospital Clínic, Barcelona, Spain

6 Head Neck Clínic, Agència de Gestió d'Ajuts Universitaris I de Recerca, 2017-SGR-01581 Barcelona, Spain 


\section{Introduction}

High-risk human papillomavirus (HPV) infection is a wellestablished risk factor for a proportion of head and neck squamous cell carcinomas, mostly located in the oropharynx [1, 2], with HPV type 16 (HPV-16) being the most frequently identified HPV genotype [3, 4].

The HPV-related oropharyngeal squamous cell carcinoma (OPSCC) has shown distinctive biology with different epidemiological, clinical, and prognostic features from HPV negative OPSCC. Particularly important are the improved response to treatment and survival of HPV-related tumors over the HPV negative tumors $[5,6]$ that lead to ongoing clinical trials of de-escalation treatments and aim to achieve good results with fewer treatment-associated comorbidities. Despite this better prognosis, around 10 to $25 \%$ of patients will suffer a disease recurrence within 5 years of treatment and another portion of patients will die from the disease [5, 7].

Thus, the determination of the HPV status is crucial to discriminate between HPV-related tumors from those which are not. Indeed, the HPV status is required in the American Joint Committee on Cancer (AJCC) 8th edition for the TNM of OPSCC staging classification [8]. For this reason, adequate detection methods for the establishment of HPV infection in head and neck carcinomas have been investigated in recent years [9-12].

The detection of HPV is based on immunohistochemical p16 expression as a surrogate marker for transcriptionally active high-risk HPV, HPV-DNA detection by in situ hybridization (ISH), HPV E6/E7 mRNA by ISH, type-specific polymerase chain reaction (PCR) techniques, and real-time PCR assays to quantify viral load [13, 14]. All these techniques are usually performed on the tumor sample biopsy or surgical specimen. However, interest in cytologic sampling is increasing due to its non-invasive, time-effective, and low-cost nature. Few studies have demonstrated the reliability and feasibility of liquid-based brush cytology specimens from oropharyngeal and oral lesions $[10,15,16]$.

Patients' management might benefit from cytology procedures not only for early diagnosis purposes but also as a followup diagnostic tool. This study aims to evaluate the usefulness of liquid-based brush cytology diagnosis and HPV detection for (1) the diagnosis of clinically suspected oropharyngeal and oral carcinomas and (2) the diagnosis of tumoral persistence after treatment for oropharyngeal or oral carcinomas.

\section{Material and methods}

\section{Patients}

This cohort study was conducted at the Pathology Department of the Hospital Clínic of Barcelona, Spain. Seventyfive patients, first diagnosed or suspected of a squamous cell carcinoma of the oropharynx or oral cavity, were included in the study between 2015 and 2018 (Fig. 1). In the Otorhinolaryngology and/or Maxillofacial Departments, brush cytology was performed and two different study groups were analyzed according to the date of the sample collection: (1) samples collected during the first endoscopy examination before treatment and (2) samples collected from the tumor site in the first control endoscopy, after treatment for squamous cell carcinoma.

After treatment, a paired cytology and biopsy were collected in lesions with high suspicion of carcinoma, whereas lesions with low suspicion were studied only by cytology.

Informed consent was signed from all patients, and the study was approved by the local ethics committee.

\section{Brush cytology samples and HPV detection}

In all cases, cytobrush heads were rotated on the lesional surface several times and transferred into a methanolbased preservative solution (ThinPrep Solution, Hologic Suisse, Lausanne, Switzerland). An aliquot of each sample was processed with liquid-based technology (ThinPrep 5000 Processor, Hologic), stained using the Papanicolaou method, and assessed under microscopy. All cases were revised by two pathologists (LA, JO) and one cytotechnologist (SA, FP, or NV) blinded to the clinical condition of the patient. Positive cytology was defined as the presence of atypical cells consistent with squamous cell carcinoma.

From another aliquot of each sample, HPV detection was performed using the Cobas HPV test (Roche Diagnostics), following published procedures [17]. This test is able to detect HPV 16, 18 and consensus high-risk HPV (31, $33,35,39,45,51,52,56,58,66,68)$. $\beta$-globin was used as a housekeeping gene. Internal controls (HPV 16, 18, high-risk consensus, and $\beta$-globin plasmids) were used on each plate. PCR consisted of 50 cycles $\left(93{ }^{\circ} \mathrm{C}\right.$ and $\left.56{ }^{\circ} \mathrm{C}\right)$ followed by $30 \mathrm{~s}$ of cooldown to $40{ }^{\circ} \mathrm{C}$ and $10 \mathrm{~s}$ at $25^{\circ} \mathrm{C}$. Data were finally assessed using LightCycler $480 \mathrm{SW}$ 1.5.0 software (Roche Diagnostics). 


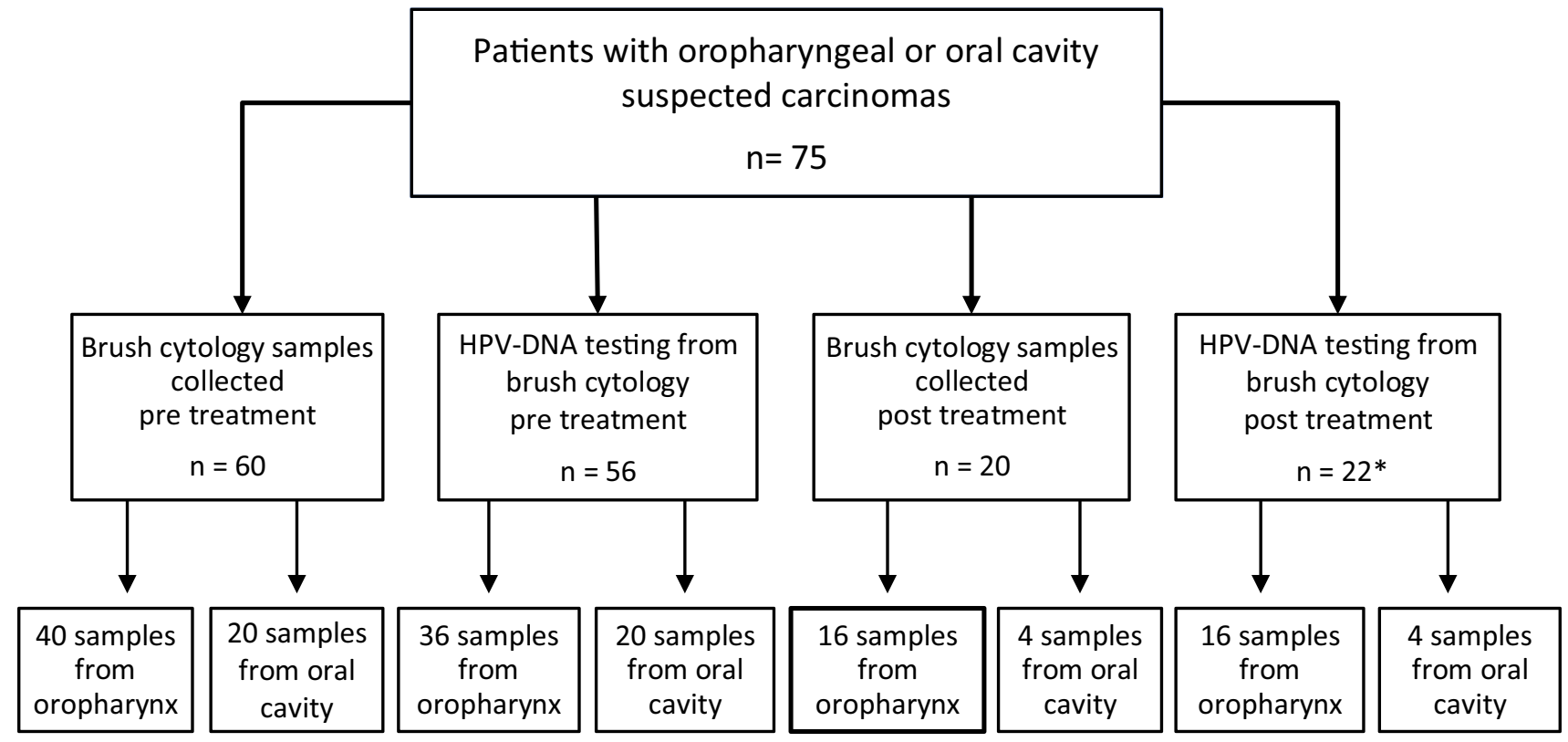

* In 2 samples the PCR result was invalid

Fig. 1 Number of cytobrush samples included in each study groups for cytology diagnosis and HPV-DNA testing

\section{Histopathological analysis, p16 immunostain, and HPV detection on tissue samples}

Biopsy of suspected lesions was performed in 60 patients. The biopsies were fixed in neutral-buffered formalin (4\%) and processed for histological analysis using standard methods. Histologic diagnosis was rendered in each case from the hematoxylin and eosin-stained tissue section, according to the World Health Organization classification for head and neck tumors [18] and revised by two pathologists (LA, PC).

p16 staining was performed from formalin-fixed and paraffin-embedded tissue sections measuring $3 \mu \mathrm{m}$ in thickness which were deparaffinized following antigen retrieval (ethylenediaminetetraacetic acid [pH 9.0] at $958 \mathrm{C}$ for $30 \mathrm{~min}$ ). p16 staining (clone E6H4 [dilution 1:10] for $30 \mathrm{~min}$; MTM Laboratories, Heidelberg, Germany) was performed on a Leica BOND MAX instrument (Leica Biosystems, Nussloch, Germany) using the Bond Polymer Refine Detection Kit (Leica). p16 positivity was defined as $\geq 70 \%$ tumor cells with strong nuclear and cytoplasmic staining [14].

Specifications on DNA extraction and HPV detection and typing are described elsewhere [19]. Briefly, HPV DNA amplification was performed using INNO-LiPA HPV Genotyping Extra II assay (INNO-LiPA; Fujirebio Europe, Ghent, Belgium). This probe assay allows the SPF10 consensus primers to amplify a $65 \mathrm{bp}$ fragment of the L1 region of the HPV genome, followed by reverse line blot hybridization to HPV type-specific immobilized probes for 32 high-risk/possibly high-risk $(16,18,25,31$, $33,35,39,45,51,52,53,56,58,59,66,68,73$, and 82 ) and 7 low-risk $(6,11,40,43,44,54$, and 70).

In all lesions, the HPV status was assessed following the established guidelines [18]. p16 was performed systematically in all samples from the oropharynx. In non-keratinizing oropharyngeal SCC, p16 positivity was considered a surrogate for transcriptionally active highrisk HPV [18]. HPV detection in tissue was performed in discordant cases between the biopsy results and the HPVDNA detection on cytology samples. In the oral cavity, outside the oropharynx, keratinizing SCCs were considered non-associated HPV carcinomas [18].

\section{Statistics}

Data were analyzed using IBM SPSS for windows version 24.0. A $p$-value of 0.05 was defined as statistically significant. To evaluate sensitivity, specificity, positive/negative predictive value, and accuracy of cytology test results after treatment, the disease was considered present (SCC persistence) when resulting in a confirmed positive biopsy, whereas no malignancy (absence of SCC persistence) was assessed by the follow-up of patients who did not show tumoral persistence or recurrence in the next 12 months. 


\section{Results}

\section{Study cohort}

From the 75 patients with a suspected carcinoma, a squamous cell carcinoma (SCC) was diagnosed in 73 cases (97\%), 72 of which were infiltrative SCC, one with in situ SCC. The remaining 2 lesions (3\%) were diagnosed with oropharynx lymphoid hyperplasia.

Figure 1 shows the number of cytobrush samples included in each study group for cytology diagnosis and HPV-DNA testing.

The demographic characteristics and tumor stage of all patients diagnosed with squamous cell carcinoma included in the study are presented in Table 1 .

\section{Value of the liquid-based brush cytology for the diagnosis of oropharyngeal and oral lesions before treatment}

Paired biopsy and cytology analysis were obtained pretreatment in 60 samples from the oropharynx $(n=40)$ and oral cavity $(n=20)$. The test showed high sensitivity and specificity (88\% and $100 \%$, respectively) and high accuracy (88\%) for the diagnosis of squamous cell carcinoma (Table 2). Disagreement between the cytology and the biopsy was considered in 5 cases where the cytology material was scarce, insufficient for diagnosis, and in 2 cases described as negative for malignancy (a total of 7 false-negative cases).

Table 1 Clinicopathological features of patients diagnosed with squamous cell carcinoma $(n=73)$ included in the study

\begin{tabular}{lll}
\hline & $\begin{array}{l}\text { Oropharyngeal } \\
\text { carcinomas }\end{array}$ & Oral cavity carcinomas \\
\hline $\begin{array}{l}\text { Total } \\
\text { Sex }\end{array}$ & $49(67.1 \%)$ & $24(32.9 \%)$ \\
Male & $39(79.6 \%)$ & $14(58.3 \%)$ \\
Female & $10(20.4 \%)$ & $10(41.6 \%)$ \\
Age (median (range)) & $63(42-92)$ & $61(47-87)$ \\
Tobacco smoke & $34(69.4 \%)$ & $16(66.7 \%)$ \\
Alcohol abuse & $24(48.9 \%)$ & $8(33.3 \%)$ \\
Stage of tumors & & $1(4.3 \%)$ \\
0 (Tis) & 0 & $2(8.7 \%)$ \\
I & $3(6.1 \%)$ & $3(13.0 \%)$ \\
II & $7(14.3 \%)$ & $2(8.7 \%)$ \\
III & $10(20.4 \%)$ & $16(65.2 \%)$ \\
IV & $28(59.2 \%)$ & \\
\hline
\end{tabular}

Table 2 Value of the cytology sample for the diagnosis of oropharyngeal and oral cavity lesions before treatment. Sensitivity, specificity, positive, negative predictive value (PPV and NPV), and accuracy

\begin{tabular}{llll}
\hline Biopsy diagnosis & Total cases & \multicolumn{2}{l}{ Cytology diagnosis } \\
\cline { 3 - 4 } & & $\begin{array}{l}\text { Positive } \\
\text { (SCC) }\end{array}$ & Negative \\
\hline SCC & 58 & 51 & 7 \\
Lymphoid hyperplasia & 2 & 0 & 2 \\
Total & 60 & 51 & 9 \\
\hline
\end{tabular}

Sensitivity: $88 \%$; specificity: 100\%; PPV: 100\%; NPV: $22 \%$; accuracy: $88 \%$

SCC squamous cell carcinoma

\section{Value of cytology samples for the HPV detection in oropharyngeal and oral lesions before treatment}

The HPV-DNA detection on cytology samples was determined in 56 samples, from the oropharynx $(n=36)$ and oral cavity $(n=20)$. The samples from the oropharynx included the 2 lymphoid hyperplasia that were negative for HPVDNA and 34 SCC. On these SCCs, HPV-DNA positivity was detected in 11 cytobrush samples $(11 / 34 ; 32 \%)$, identifying an HPV16 genotype in all but one sample. A discrepancy was observed in two non-keratinizing SCCs (2/34; $6 \%$ ), in which the p16 immunostain in the tissue sample was positive, whereas the HPV-DNA detection in the cytobrush sample was negative. In both cases, HPV-DNA testing was re-conducted in the tissue samples showing positivity for HPV 16 in the further analysis. Thus, the HPV detection using cytobrush samples from oropharyngeal squamous cell

Table 3 Value of the HPV detection on cytology samples from oropharyngeal squamous cell carcinomas before treatment. Sensitivity, specificity, positive, negative predictive value (PPV and NPV), and accuracy

\begin{tabular}{llll}
\hline $\begin{array}{l}\text { HPV status according to } \\
\text { the biopsy sample }\end{array}$ & Total cases & $\begin{array}{l}\text { Cytology HPV detec- } \\
\text { tion }\end{array}$ \\
\cline { 3 - 4 } & & HPV + & HPV - \\
\hline Positive & 13 & 11 & 2 \\
Negative & 21 & 0 & 21 \\
Total & 34 & 11 & 23 \\
\hline
\end{tabular}

Sensitivity: $85 \%$; specificity: 100\%; PPV: $100 \%$; NPV: $91 \%$; accuracy: $91 \%$

$H P V$ human papilloma virus

*HPV status: HPV-associated squamous cell carcinoma (SCC) showed non-keratinizing morphology and p16 positive immunostain. In the two discordant cases with the HPV-DNA detection on cytology, HPV positivity was confirmed with HPV-DNA detection in tissue 
carcinoma showed $85 \%$ sensitivity, $100 \%$ specificity, and $91 \%$ accuracy (Table 3 ).

All 20 squamous cell carcinomas from the oral cavity showed HPV-DNA negativity, according to a keratinizing SCC morphology and location outside the oropharynx.

\section{Value of the liquid-based brush cytology for the diagnosis of oropharyngeal and oral carcinoma persistence after treatment}

During the follow-up after treatment, brush cytology was assessed in a total of 20 samples from the oropharynx $(n=16)$ and oral cavity $(n=4)$ tumor site. Patients had been treated with surgery $(n=3)$, radiotherapy alone $(n=4)$, and/

Table 4 Value of the cytology sample for the diagnosis of oropharyngeal and oral cavity lesions after treatment. Sensitivity, specificity, positive, negative predictive value (PPV and NPV), and accuracy

\begin{tabular}{llll}
\hline Diagnosis & Total cases & \multicolumn{2}{l}{ Cytology diagnosis } \\
\cline { 3 - 4 } & & $\begin{array}{l}\text { Positive } \\
\text { (SCC) }\end{array}$ & Negative \\
\hline SCC persistence & 7 & 5 & 2 \\
No malignancy & 13 & 3 & 10 \\
Total & 20 & 8 & 12 \\
\hline
\end{tabular}

Sensitivity: 71.4\%; specificity: $76.9 \%$; PPV: $63 \%$; NPV: $83 \%$; accuracy: $75 \%$

SCC squamous cell carcinoma

SCC persistence: squamous cell carcinoma persistence was confirmed by positive biopsies. No malignancy: the absence of SCC was assessed by the follow-up of patients who did not show tumoral persistence or recurrence or a combination with chemotherapy $(n=13)$. The samples were collected from 36 to 249 (median 136) days after finishing treatment.

The lesions were studied by cytology and biopsy simultaneously in 9 cases showing high clinical suspicion for carcinoma and only cytology in 11 showing low suspicion of malignancy. The overall results of the cytology after treatment (patients with concomitant biopsy or long follow-up) showed a sensitivity of $71.4 \%$, specificity of $76.9 \%$, and accuracy of $75 \%$ (Table 4 ). The discrepancies were observed in 5 cases: 2 false-negative results for malignancy and 3 false-positive results that showed atypical cells attributed to post-treatment reactive changes (Fig. 2).

\section{Value of cytology samples for the HPV detection in oropharyngeal and oral sites after treatment}

HPV-DNA detection was available in 22 cytobrushes performed after treatment, demonstrating 19 HPV-DNA negative cases, and one case HPV16-DNA positive. The HPVpositive case corresponded to an HPV-related SCC treated with chemotherapy and radiotherapy with tumoral persistence 3 months after treatment, confirmed by biopsy and cytology.

The 19 HPV negative results were consistent with the HPV expected status after treatment: 8 oropharynx HPVassociated SCC with no tumoral persistence after treatment and 11 SCC that were HPV-non associated SCC before treatment (7 oropharynx SCC and 4 oral cavity SCC). Thus, the HPV detection using cytobrush samples showed $100 \%$ sensitivity, specificity, and accuracy (Table 5).

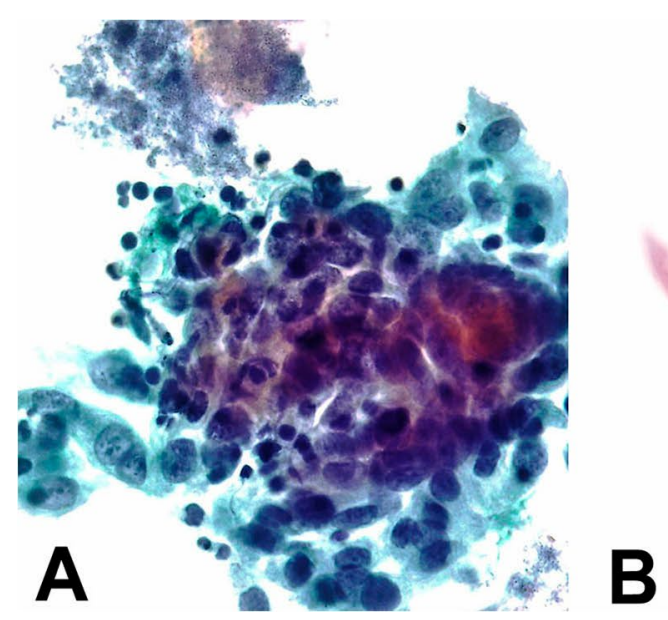

Fig. 2 Examples of cytologic specimens from oropharyngeal and oral cavity squamous cell carcinomas: a atypical cells from an oropharyngeal HPV-related squamous cell carcinoma, before treatment; b atypical cells from an oral cavity squamous cell carcinoma, before treatment; c atypical cells from the oropharynx in a patient with-

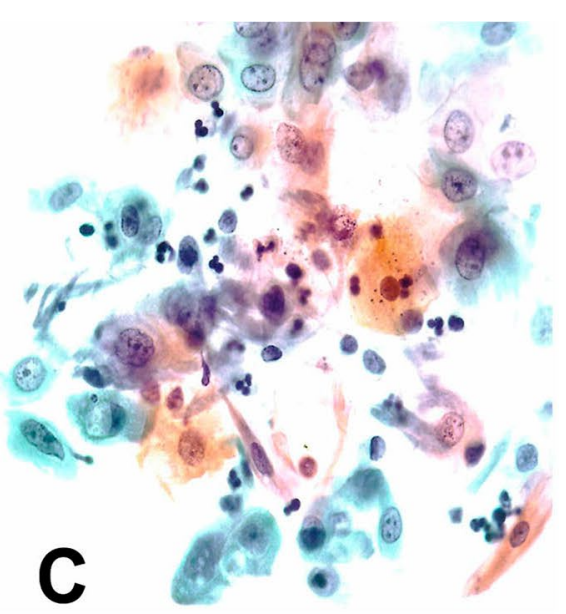

out tumoral persistence, attributed to reactive changes secondary to received treatment. Note the similarities within the three samples, all showing high nucleus/cytoplasm ratio, visible and irregularly dispersed nucleoli, and atypical chromatin with nuclear clearing. Elongated, spindle-shaped cells are observed in samples B and C 
Table 5 Value of the HPV detection on cytology samples from oropharyngeal and oral cavity lesions after treatment. Sensitivity, specificity, positive, negative predictive value (PPV and NPV), and accuracy

\begin{tabular}{llll}
\hline $\begin{array}{l}\text { HPV expected status } \\
\text { after treatment } *\end{array}$ & Total cases & $\begin{array}{l}\text { Cytology } \\
\text { HPV }+\end{array}$ & $\begin{array}{l}\text { HPV detection } \\
\text { HPV }-\end{array}$ \\
\hline HPV positive & 1 & 1 & 0 \\
HPV negative & 19 & 0 & 19 \\
Total & 20 & 1 & 19 \\
\hline
\end{tabular}

Sensitivity: 100\%; specificity: 100\%; PPV: 100\%; NPV: 100\%; accuracy: $100 \%$

$H P V$ human papilloma virus

*HPV expected status: HPV positive: SCC-HPV-associated persistence. HPV negative: SCC-HPV-associated without SCC persistence and non-associated HPV SCC before treatment

In two cases $(2 / 22 ; 9 \%)$, the cytobrush sample had insufficient DNA quality or quantity for testing, leading to invalid results.

\section{Discussion}

To the best of our knowledge, this is the first study to assess the usefulness of both liquid-based cytology and HPV detection in patients with oropharyngeal and oral cancer before and after treatment. Only Kofler et al. have also evaluated post-treatment samples but only from the oropharynx and for HPV determination [20].

According to our results, the study of morphology on liquid-based cytology obtained by cytobrush before and after treatment may give equivocal results for squamous cell carcinoma diagnosis, although it can be of great utility for the detection of HPV-DNA.

The use of cytobrush for further liquid-based cytology is a well-tolerated and minimally invasive technique for collecting samples [16, 21]; however, a systematic review by Alsarraf et al. indicates variable results without clear recommendations on the effectiveness of this technique for the diagnosis of lesions on the head and neck [22]. In this regard, our cytology results from samples performed before treatment, when compared with the biopsy (gold standard), showed a high sensitivity (over $80 \%$ ) and specificity (100\%). The sensitivity results are in line with published data by Donà et al., with 164 patients who showed a sensitivity of $75.5 \%$, although showing a low specificity. The high specificity observed in our series might be related to the chosen methodology for the cytomorphology evaluation. In our study, the presence of "atypical cells" was classified as positive for malignancy. A larger cohort, although limited to oral samples, has shown a specificity rising to $84.9 \%$ [21], also similar to our results. A plausible explanation for the false-negative results found in our study could be the inaccessibility of the lesions. Anatomical limitations particularly in the oropharynx, in which SCC can develop in the tonsillar crypts, are likely a limiting factor in sampling with cytobrush [23, 24].

In addition, we evaluated the value of the cytomorphology for the diagnosis after treatment. To that purpose, the cytology results were evaluated against the persistence or not of SCC, assessed by biopsy or by the follow-up of the patients. To our understanding, to date, no data is rendered on the effectiveness of this technique in samples taken posttreatment since most authors center on the cytology utility as a screening test or for initial diagnosis and not for follow-up $[22,25]$. Our results indicate a moderate accuracy $(75 \%)$, sensitivity $(71.4 \%)$, and specificity $(76.9 \%)$ for cytology diagnosis in these samples. Its low accuracy makes this technique less effective when used alone to monitor patients after treatment. The false-positive cases observed in our study showed "atypical" cells, most likely an overinterpretation of reparative changes as a consequence of the treatment received, representing a barrier when evaluating cytomorphology. Moreover, cytologic abnormalities in oropharyngeal brushings and oral rinses can be associated with smoking and drinking habits $[22,26]$.

Concerning HPV detection, our results correlate with several studies confirming that cytobrush samples are useful in determining HPV status with the Roche Cobas HPV test [7, 27, 28]. Before treatment, samples were evaluated against the p16 immunostain or tissue HPV-DNA detection. For this reason, the analysis was done only in oropharyngeal samples where the immunostain was done routinely as a surrogate marker. However, the absence of HPV detection in all oral SCC tested correlates with the keratinizing morphology and location outside the oropharynx, following the established clinical guides and our experience [18, 29]. Our results support the HPV detection in cytobrush samples as a valid and reliable alternative method for the assessment of HPV in oropharyngeal and oral carcinoma. Access to easy but robust methods is particularly necessary, with increasing data indicating the need for double testing for HPV since double positivity for HPV-DNA/p16INK4a showed the strongest diagnostic accuracy and prognostic value [30]. The stratification of the patients could rise in importance as de-escalation strategies will be finally established. Although several ongoing clinical trials are aiming to reduce treatment-associated toxicities without affecting the superior survival rates, there is still a lack of strong evidence to currently recommend any of the chemotherapy schemes proposed for de-escalation treatment [31].

In our study, erroneous stratification would be performed if HPV status was based in one method alone. In 2 HPV-associated oropharyngeal carcinomas (6\%), HPVDNA was not detected in the cytology sample. In addition, 
the HPV status based only on the p16 immunostain could falsely classify the patients as HPV positive or negative, according to the results in the literature, ranging from 4.9 to $26.2 \%$ [32, 33]

The validation of methods for the correct diagnosis of HPV status in post-treated patients is crucial for prognosis and surveillance. Our results showed that HPV determination in samples after treatment successfully correlates with the HPV expected status post-treatment with an accuracy of $100 \%$. In our series, only one patient with HPV16related tumor showed tumoral persistence, which correlates with the HPV-DNA positivity in the post-treatment sample. As shown in the data published by Kofler et al., the same genotype as that identified in the pre-treatment analysis was identified in the HPV-positive post-treatment sample. In our study, HPV-associated OPSCC with an HPV negative result after treatment correlated with no tumoral persistence, in agreement with Kofler et al. results [20]. Nevertheless, we have to take into account that in up to $9 \%$ of the cohort the test was invalid, attributed to the scarce number of cells and insufficient DNA detection in these samples.

Importantly, the selection of cytobrush procedure instead of using oral rinses for the extraction of the samples might play a part in the good results observed in our study. Some previous studies have compared the performance of cytobrush samples vs oral rinses samples for HPV determination and found poor agreement between the two methods [34] and stated that oral rinse cytology showed lower sensitivity (43-88\%) for the assessment of HPV infection [35]. Indeed, technical problems were identified during the study as scarce cells were identified in some cases which led to false-negative results. To overcome this technical issue, the collection of the samples should be obtained by performing several passes of the cytobrush in the suspected area. Another limitation of our study includes the sample size, particularly in the group of patients with paired biopsy and cytology after treatment.

Due to ethical issues, patients with low suspicion of carcinoma persistence were not biopsied. However, a long follow-up of the patients confirmed the absence of tumoral persistence after treatment in this group of patients. In addition, in our series, only squamous cell carcinomas were evaluated, so the render of cytology in dysplastic lesions was not assessed in our study. To properly address cytology accuracy for diagnosis, the tested samples should comprise more negative samples.

All results considered, this study contributes to raising the evidence on the effectiveness of cytobrush for establishing the HPV status in oral and oropharyngeal carcinomas, which could improve the diagnostic approaches and algorithms. This study also confirms the usefulness of these samples to rule out HPV-associated oropharyngeal carcinoma persistence after treatment, as a potential method to monitor the patients.

\section{Conclusions}

Brush cytology samples are reliable for the detection of HPV DNA before and after treatment and may be a useful method to incorporate in the HPV testing guidelines. Brush cytology morphology showed fair results for the diagnosis of SCC before and after treatment. Further analysis on a larger patient cohort is necessary to confirm our results.

Author contribution LA, SA, and JO designed the study; JO, IV, AF, $\mathrm{CM}$, and SA collected data and organized the database; JO, FP, NV, SA, PC, and LA performed the tests; PC, JO, FP, and LA analyzed and interpreted data; $\mathrm{PC}$ and $\mathrm{JO}$ performed the statistical analysis; $\mathrm{PC}$ and JO wrote the first draft of the manuscript; all authors contributed to manuscript revision, read, and approved the submitted version.

Funding Open Access funding provided thanks to the CRUE-CSIC agreement with Springer Nature. This study was funded by Premio Emili Letang of the Hospital Clinic of Barcelona.

\section{Declarations}

Ethics approval The questionnaire and methodology for this study was approved by the Human Research Ethics committee of the Hospital Clinic of Barcelona (Ethics approval number: 2011/6523.).

Informed consent Informed consent was obtained from all individual participants included in the study.

Conflict of interest The authors declare no competing interests.

Open Access This article is licensed under a Creative Commons Attribution 4.0 International License, which permits use, sharing, adaptation, distribution and reproduction in any medium or format, as long as you give appropriate credit to the original author(s) and the source, provide a link to the Creative Commons licence, and indicate if changes were made. The images or other third party material in this article are included in the article's Creative Commons licence, unless indicated otherwise in a credit line to the material. If material is not included in the article's Creative Commons licence and your intended use is not permitted by statutory regulation or exceeds the permitted use, you will need to obtain permission directly from the copyright holder. To view a copy of this licence, visit http://creativecommons.org/licenses/by/4.0/.

\section{References}

1. Gillison ML (2000) Evidence for a causal association between human papillomavirus and a subset of head and neck cancers. J Natl Cancer Inst 92(9):709-720

2. Leemans CR, Snijders PJF, Brakenhoff RH (2018) The molecular landscape of head and neck cancer. Nat Rev Cancer 18(5):269-282

3. Tumban E (2019) A current update on human papillomavirusassociated head and neck cancers. Viruses 11(10):922 
4. Ndiaye C, Mena M, Alemany L, Arbyn M, Castellsagué X, Laporte L et al (2014) HPV DNA, E6/E7 mRNA, and p16INK4a detection in head and neck cancers: a systematic review and metaanalysis. Lancet Oncol 15(12):1319-1331

5. Ang KK, Harris J, Wheeler R, Weber R, Rosenthal DI, NguyenTân PF et al (2010) Human papillomavirus and survival of patients with oropharyngeal cancer. N Engl J Med 363(1):24-35

6. Posner MR, Lorch JH, Goloubeva O, Tan M, Schumaker LM, Sarlis NJ et al (2011) Survival and human papillomavirus in oropharynx cancer in TAX 324: a subset analysis from an international phase III trial. Ann Oncol 22(5):1071-1077

7. Du E, Mazul AL, Farquhar D, Brennan P, Anantharaman D, Abedi-Ardekani B et al (2019) Long-term survival in head and neck cancer: impact of site, stage, smoking, and human papillomavirus status. Laryngoscope 129(11):2506-2513

8. Amin MB, American Joint Committee on Cancer, American Cancer Society, editors. AJCC cancer staging manual. Eight edition / editor-in-chief, Mahul B. Amin, MD, FCAP ; editors, Stephen B. Edge, MD, FACS [and 16 others] ; Donna M. Gress, RHIT, CTR-Technical editor ; Laura R. Meyer, CAPM-Managing editor. Chicago IL: American Joint Committee on Cancer, Springer; 2017. 1024 p.

9. Bishop JA, Maleki Z, Valsamakis A, Ogawa T, Chang X, WestraPai SI et al (2012) Application of the hybrid capture 2 assay to squamous cell carcinomas of the head and neck: a convenient liquid-phase approach for the reliable determination of human papillomavirus status. Cancer Cytopathol 120(1):18-25

10. Broglie MA, Jochum W, Förbs D, Schönegg R, Stoeckli SJ (2015) Brush cytology for the detection of high-risk HPV infection in oropharyngeal squamous cell carcinoma: brush cytology for HPV testing in OPSCC. Cancer Cytopathol 123(12):732-738

11. Krane JF (2013) Role of cytology in the diagnosis and management of HPV-associated head and neck carcinoma. Acta Cytol 57(2):117-126

12. Chera BS, Kumar S, Shen C, Amdur R, Dagan R, Green R et al (2020) Plasma circulating tumor HPV DNA for the surveillance of cancer recurrence in HPV-associated oropharyngeal cancer. JCO 38(10):1050-1058

13. Larque AB, Hakim S, Ordi J, Nadal A, Diaz A, del Pino M et al (2014) High-risk human papillomavirus is transcriptionally active in a subset of sinonasal squamous cell carcinomas. Mod Pathol 27(3):343-351

14. Lewis JS, Chernock RD, Ma X-J, Flanagan JJ, Luo Y, Gao G et al (2012) Partial p16 staining in oropharyngeal squamous cell carcinoma: extent and pattern correlate with human papillomavirus RNA status. Mod Pathol 25(9):1212-1220

15. Donà MG, Giuliani M, Vocaturo A, Spriano G, Pichi B, Rollo F et al (2014) Cytology and human papillomavirus testing on cytobrushing samples from patients with head and neck squamous cell carcinoma: cytology and HPV in head and neck pathology. Cancer 120(22):3477-3484

16. Kujan O, Pemberton MN, Schwarz M, Sloan P (2018) Evaluation of an innovative oral brush for potential applications using liquid based cytology. J Oral Sci 60(1):45-50

17. Wright MF, Weiss VL, Lewis JS, Schmitz JE, Ely KA (2020) Determination of high-risk HPV status of head and neck squamous cell carcinoma using the Roche cobas HPV test on cytologic specimens and acellular supernatant fluid. Cancer Cytopathol 128(7):482-490

18. El-Naggar AK, Chan JKC, Grandis JR, Takata T, Slootweg PJ, editors. WHO classification of head and neck tumours. 4th edition. Lyon: International Agency for Research on Cancer; 2017. 347 p. (World Health Organization classification of tumours).
19. Alos L, Moyano S, Nadal A, Alobid I, Blanch JL, Ayala E et al (2009) Human papillomaviruses are identified in a subgroup of sinonasal squamous cell carcinomas with favorable outcome. Cancer 115(12):2701-2709

20. Kofler B, Borena W, Dudas J, Innerhofer V, Dejaco D, Steinbichler TB et al (2020) Post-treatment HPV surface brushings and risk of relapse in oropharyngeal carcinoma. Cancers 12(5):1069

21. Deuerling, Gaida, Neumann, Remmerbach. Evaluation of the accuracy of liquid-based oral brush cytology in screening for oral squamous cell carcinoma. Cancers. 2019;11(11):1813.

22. Alsarraf HA, Kujan O, Farah CS (2018) The utility of oral brush cytology in the early detection of oral cancer and oral potentially malignant disorders: a systematic review. J Oral Pathol Med. 47(2):104-16

23. Lingen MW (2011) Brush-based cytology screening in the tonsils and cervix: there is a difference! Cancer Prev Res 4(9):1350-1352

24. Remacha J, Castillo PVI (2021) Metastatic cervical carcinoma of unknow primary. Acta Otorrinolaringol Esp S001-6519(21):00036-00044

25. Kujan O, Glenny A-M, Oliver R, Thakker N, Sloan P. Screening programmes for the early detection and prevention of oral cancer. In: The Cochrane Collaboration, editor. Cochrane Database of Systematic Reviews [Internet]. Chichester, UK: John Wiley \& Sons, Ltd; 2006 [cited 2021 Jul 1]. p. CD004150.pub2. Available from: https://onlinelibrary.wiley.com/doi/10.1002/14651858. CD004150.pub2

26. Benevolo M, Rollo F, Giuliani M, Pichi B, Latini A, Pellini R et al (2020) Abnormal cytology in oropharyngeal brushings and in oral rinses is not associated with HPV infection: the OHMAR study. Cancer Cytopathol 128(9):648-655

27. Kerr DA, Pitman MB, Sweeney B, Arpin RN, Wilbur DC, Faquin WC (2014) Performance of the Roche cobas 4800 high-risk human papillomavirus test in cytologic preparations of squamous cell carcinoma of the head and neck: Cobas Test for HR-HPV in Head \& Neck SCC. Cancer Cytopathol 122(3):167-174

28. Harlé A, Guillet J, Thomas J, Sastre-Garau X, Rouyer M, Ramacci C et al (2018) Evaluation and validation of HPV real-time PCR assay for the detection of HPV DNA in oral cytobrush and FFPE samples. Sci Rep 8(1):11313

29. de la Oliva J, Larque A-B, Marti C, Bodalo-Torruella M, Nonell L, Nadal A, et al. Oral premalignant lesions of smokers and nonsmokers show similar carcinogenic pathways and outcomes. A clinicopathological and molecular comparative analysis. J Oral Pathol Med. 2021;50(3):280-6.

30. Mena M, Taberna M, Tous S, Marquez S, Clavero O, Quiros B et al (2018) Double positivity for HPV-DNA/p16ink4a is the biomarker with strongest diagnostic accuracy and prognostic value for human papillomavirus related oropharyngeal cancer patients. Oral Oncol 78(February):137-144

31. Rühle A, Grosu A-L, Nicolay NH (2021) De-escalation strategies of (chemo)radiation for head-and-neck squamous cell cancersHPV and beyond. Cancers 13(9):2204

32. Shinn JR, Davis SJ, Lang-Kuhs KA, Rohde S, Wang X, Liu P et al (2021) Oropharyngeal squamous cell carcinoma with discordant p16 and HPV mRNA results: incidence and characterization in a large, contemporary United States cohort. Am J Surg Pathol. https://journals.lww.com/10.1097/PAS.0000000000001685

33. Quabius ES, Tribius S, Heinrichs A, Haaser D, Kühnel A, Laudien $M$ et al (2021) HPV DNA/RNA detection in various oral and oropharyngeal biomaterials identifies active HPV infections also in non-neoplastic tonsils. Translational Oncology 14(2):101002

34. Donà MG, Pichi B, Rollo F, Benevolo M, Latini A, Laquintana V et al (2019) Human papillomavirus detection in matched oral 
rinses, oropharyngeal and oral brushings of cancer-free high-risk individuals. Oral Oncol 91(February):1-6

35. D'Souza G, Clemens G, Troy T, Castillo RG, Struijk L, Waterboer $T$ et al (2019) Evaluating the utility and prevalence of HPV biomarkers in oral rinses and serology for HPV-related oropharyngeal cancer. Cancer Prev Res 12(10):689-699
Publisher's Note Springer Nature remains neutral with regard to jurisdictional claims in published maps and institutional affiliations. 